\title{
COVID-19 pneumonia following double-sleeve lobectomy for lung cancer
}

\author{
Yusuf Inanc ${ }^{1} \cdot$ Guven Olgac $^{2} \cdot$ Merih Kalamanoglu Balci $^{3} \cdot$ Cemal Asim Kutlu $^{1}$
}

Received: 12 July 2020 / Accepted: 21 September 2020 / Published online: 30 September 2020

(c) The Japanese Association for Thoracic Surgery 2020

\begin{abstract}
Here, we report a 54-year-old man who underwent double-sleeve left upper lobectomy for lung cancer and his postoperative course was complicated with COVID-19 pneumonia. Five days after his discharge from hospital, he was re-admitted with mild fever and bilateral multiple ground glass opacities on his chest CT. PCR testing confirmed COVID-19 infection and he was treated according to policies established by our nation's health authority. He is still receiving adjuvant chemotherapy and remains well at 3 months after the operation.
\end{abstract}

Keywords Double sleeve lobectomy · Postoperative complication · COVID-19 pneumonia

$\begin{array}{ll}\text { Abbreviations } \\ \text { COVID-19 } & \text { Coronavirus disease 2019 } \\ \text { CT } & \text { Computed tomography } \\ \text { FDG } & \text { Fluoro-deoxy glucose } \\ \text { GGO } & \text { Ground glass opacity } \\ \text { NSCLC } & \text { Non-small-cell lung cancer } \\ \text { PCR } & \text { Polymerase chain reaction } \\ \text { PET } & \text { Positron emission tomography } \\ \text { SARS-CoV-2 } & \text { Severe acute respiratory syndrome corona- } \\ & \text { virus 2 } \\ \text { VATS } & \text { Video assisted thoracic surgery }\end{array}$

\section{Introduction}

Dreadful spreading features of the COVID-19 pandemic have rapidly extinguished healthcare sources of many countries regardless of their development level. This has greatly influenced the treatment algorithms of lung cancer with

Yusuf Inanc

yusufinanc.md@gmail.com

1 Department of Thoracic Surgery, Bahcesehir University School of Medicine, Merdivenköy, 23 Nisan Sok. No:17, Kadıköy, 34732 Istanbul, Turkey

2 Department of Thoracic Surgery, Yedikule Chest Diseases and Thoracic Surgery Research and Teaching Hospital, Istanbul, Turkey

3 Department of Pulmonology, Bahcesehir University School of Medicine, Istanbul, Turkey resultant decrease in number of elective surgeries as hospital admissions of COVID-19 cases have increased [1, 2]. It has, in turn, forced thoracic surgeons to prioritize surgical referrals more responsibly according to their individualized stage-based treatment needs [1-3]. In this respect, decisionmaking process of each patient became a challenging one as many of them are susceptible to this infection throughout the peri-operative period.

Considering that postoperative outcome and survival rates are significantly better in patients undergoing lung sparing procedures for NSCLC and unnecessarily extended resections almost invariably lead to detrimental consequences [4], every effort should devotedly be made to preserve as much lung tissue as possible. Here, we report such a case who underwent double-sleeve left upper lobectomy during the early days of COVID-19 pandemic in Turkey.

\section{Case}

A 54-year-old male was presented with unproductive cough lasting for 4 weeks. Physical examination was otherwise normal, but CT of chest revealed a $6 \mathrm{~cm}$ lobulated mass surrounding the left upper lobe bronchus and possibly invading the left main pulmonary artery. At bronchoscopy, a tumor originating from the left upper lobe orifice and invading the secondary carina was observed. Biopsies revealed a squamous cell carcinoma. PET scan did not discover N2 disease or distant metastasis. After confirming that he would tolerate even a left pneumonectomy, we 
proceeded to lung resection in an attempt to perform a double sleeve left upper lobectomy.

At operation, initial exploration was done with uni-portal VATS approach. At a glance, it soon became apparent that the safe preparation and proximal control of the left pulmonary artery was not possible due to proximal tumor extension. Thus, the incision was converted to a lateral thoracotomy. The preparation and the insertion of a vascular clamp to the very proximal part of the left pulmonary artery could have only been achieved intrapericardially after dividing the ligamentum arteriosum. Division of the superior pulmonary vein and subsequent sleeve resections of both upper lobe bronchus and a segment of the proximal pulmonary artery followed by systematic lymph node dissection was straightforward, so was the bronchial anastomosis after confirmation of clear bronchial resection margins on frozen section. However, arterial anastomosis could be accomplished with extreme difficulty requiring permissive bleeding. The postoperative course was uneventful afterwards, and the patient was discharged home on 5th postoperative day. Histopathology was reported as squamous cell carcinoma invading the left main pulmonary artery along with \#11S and \#12 intrapulmonary lymph nodes (pT4N1M0; pStage IIIA) and clear resection margins.

The patient was re-admitted to the ward with resisting mild fever $\left(37.9^{\circ} \mathrm{C}\right)$, unproductive cough and dyspnea on 10th postoperative day. Because of increasing incidence of COVID-19 cases in our country, he underwent CT of the chest and PCR testing of oropharyngeal and nasal swabs. CT revealed multiple bilateral GGOs that were compatible with COVID-19 pneumonia (Fig. 1). Diagnosis was confirmed with a positive PCR testing and treatment according to our national guidelines was initiated. This included Hydroxychloroquine, Oseltamivir and Azithromycin with addition of Piperacillin/Tazobactam on 3rd day because, bacterial pneumonia as a superinfection could not be excluded. Due to persistent fever, increasing dyspnea and the emerging need for oxygen support, Oseltamivir and Azithromycin were stopped and Favipiravir was started on the 6th day of treatment. However, he was found to be developing some degree of pulmonary fibrosis on repeat CT of the chest by the 10th day of initial treatment, thus, a 3-day course of Prednisolone was given at a daily dose of $40 \mathrm{mg}$. His clinical status improved gradually and he was discharged home on the 12th day of anti-COVID-19 treatment, provided he quarantines himself at home for 2 weeks. Before referring him to oncology clinic for adjuvant chemotherapy, we confirmed his complete recovery from COVID-19 infection with three subsequent negative PCR testing, near total clearance of lung lesions on CT scan and the presence of acquired neutralizing antibodies against SARS-CoV-2 in his serum.
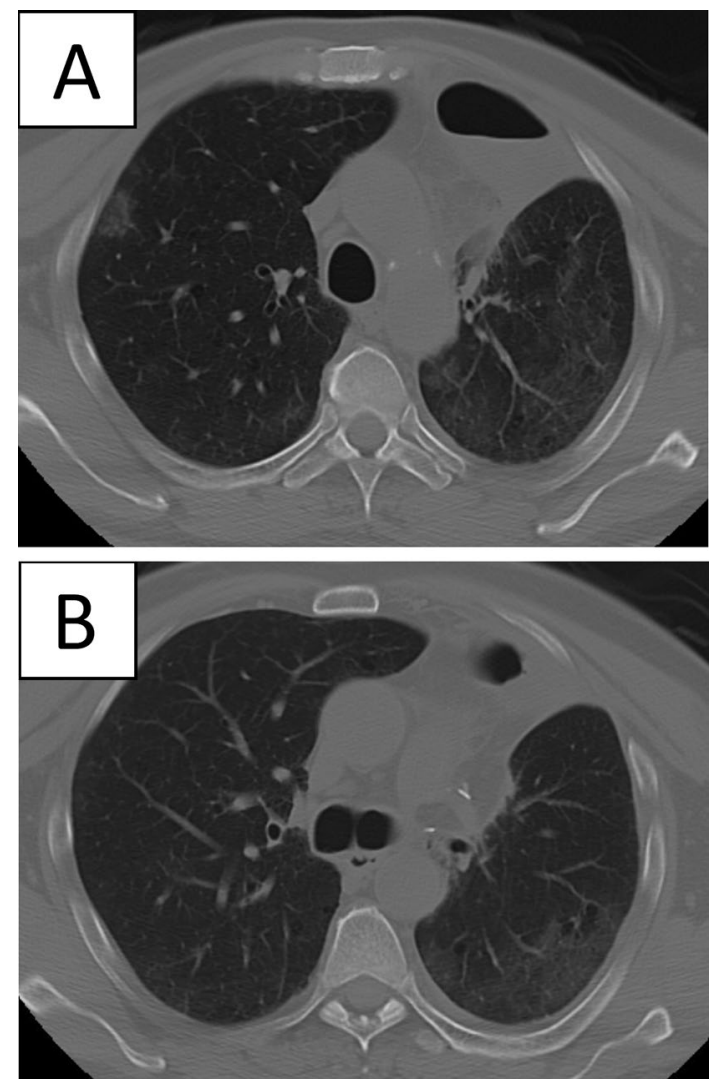

Fig. 1 GGO's on both sides related to COVID pneumonia (a and b), the PA anastomosis with nearby surgical clips (b)

\section{Comments}

COVID-19 pandemic had a significant effect on the organization of elective surgical procedures soon after it has emerged as a serious healthcare issue in many countries [1-3]. Then, in the shortest time possible, healthcare professionals and medical societies declared the preoperative cautions and required investigations for elective surgery $[2,3,5]$. Delicate policies have also been proposed to protect non-COVID patients from being destitute of receiving their necessary treatments for other demanding health issues, especially those of developing as an emergency [5].

Because our patient was asymptomatic at the time of referral, we screened him with PCR test only and it failed to discover SARS-CoV-2 infection preoperatively. This operation was also performed in the very early days of the COVID-19 pandemic in our country, and the utility of preoperative CT scanning prior to elective cancer surgery was controversial in those days [6]. As our case was asymptomatic and his last CT was obtained only 16 days before the operation without any evidence of SARS-CoV-2 infection, we did not repeat it. Meanwhile, regularly updated guidelines by a collaborative group from Great Britain and 
Ireland [7] was also in favor of our approach. Thus, we still evaluate all our thoracic surgical candidates, accordingly. This approach denies repeating CT of the chest, but necessitates consecutive two PCR testing of oropharyngeal and nasal swabs-the last one being within $72 \mathrm{~h}$ of planned operation-provided that they were asymptomatic and were also self-isolated themselves for the last 14 days. Consequently, it is impossible to know if our patient had the infection beforehand or he has got infected during the perioperative course. Unfortunate enough, however, a member of the surgical team (scrub nurse) was also found to be COVID-19 positive on PCR testing, 2 weeks after the operation. The same dilemma is valid for her, and we will never know the source of her SARS-CoV-2 infection.

As postoperative CT scan determined that GGOs were more dominant on the operated side, we may speculate that division of the bronchus and re-anastomosis might have adversely affected the clearance of bronchial secretions owing to impaired bronchial ciliary motility. Like any other postoperative infectious processes, our patient would have faced detrimental consequences of a pneumonectomy during the course of his COVID-19 pneumonia if a lung sparing resection was not performed. As a result, this ensured us to manage his COVID-19 pneumonia relatively smoothly without the need for a more aggressive respiratory support. On the other hand, however, this postoperative complication caused nearly 4 week delay in the initiation of adjuvant chemotherapy for his T4N1 disease.

\section{Conclusion}

To conclude, in addition to other obligatory measures, we must pay more attention and try to force ourselves further than ever to perform lung sparing resections for the surgical treatment of lung cancer to prevent our patients from catastrophic respiratory consequences associated with COVID19 disease and other respiratory failure-related co-morbidities during this pandemic.
Acknowledgements We would like to thank Ms. Ezgi June Olgac, BSc. for her invaluable contribution in proofreading of the manuscript.

\section{References}

1. Thoracic surgery at the time of COVID-19: a 27 Q\&A snapshot on behalf of the ESTS Steering Learning Affairs Committee. Results of the ESTS online survey on COVID-19 pandemic and impact on thoracic oncologic surgical activity. Available at: https://www. ests.org/_userfiles/pages/files/COVID19_ESTS_APRIL2020-min. pdf (Accessed 26 May 2020)

2. Liang W, Guan W, Chen R, et al. Cancer patients in SARSCoV-2 infection: a nationwide analysis in China. Lancet Oncol. 2020;21(3):335-7.

3. Thoracic Surgery Outcomes Research Network Inc. COVID-19 Guidance for triage of operations for thoracic malignancies: a consensus statement from thoracic surgery outcomes research network. Ann Thora Surg. 2020. https://doi.org/10.1016/j.athor acsur.2020.03.005.

4. Gomez-Caro A, Garcia S, Reguart N, et al. Determining the appropriate sleeve lobectomy versus pneumonectomy ratio in central non-small cell lung cancer patients: an audit of an aggressive policy of pneumonectomy avoidance. Eur J Cardiothorac Surg. 2011;39:352-9.

5. COVID-19: Elective Case Triage guidelines for surgical care. American College of Surgeons. STS COVID-19 Resources, surgical triage. Available at: https://www.facs.org/covid-19/clinicalguidance/elective-case/thoracic-cancer. (Accessed May 26, 2020)

6. Callaway M, Harden S, Ramsden W, et al. A national UK audit for diagnostic accuracy of preoperative CT chest in emergency and elective surgery during COVID-19 pandemic. Clin Radiol. 2020;75(9):705-8.

7. Guidelines for pre-operative COVID-19 testing for elective cancer surgery. https://www.rcsed.ac.uk/media/681195/guidelines -for-pre-operative-covid-19-testing-for-elective-cancer-surge ry-1305202.pdf?utm_source $=$ RCR+Staff+-+ebulletins\&utm campaign $=615 \mathrm{e} 6 \mathrm{~d} 9 \mathrm{ea} 9$-Coronavirus_be_alert_140520_staff \&utm_medium =email\&utm_term=0_582a53c738-615e6d9ea9 -87141553 (Accessed 31 Aug 2020)

Publisher's Note Springer Nature remains neutral with regard to jurisdictional claims in published maps and institutional affiliations. 